\title{
Power System Stabilizer Design Using Compressed Rule Base of Fuzzy Logic Controller
}

\author{
Dhanesh Kumar Sambariya \\ Department of Electrical Engineering, Rajasthan Technical University, Kota, India
}

Email address:

dsambariya_2003@yahoo.com

\section{To cite this article:}

Dhanesh Kumar Sambariya. Power System Stabilizer Design Using Compressed Rule Base of Fuzzy Logic Controller. Journal of Electrical and Electronic Engineering. Vol. 3, No. 3, 2015, pp. 52-64. doi: 10.11648/j.jeee.20150303.16

\begin{abstract}
In this paper, the fuzzy logic controller (FLC) based power system stabilizer (PSS) with compressed / reduced rule is presented. The FLC rule base is generally based on empirical control rules. In this method, the fuzzy system with a large number of fuzzy rules is compressed to a fuzzy system with a reduced number of rules by removing the redundant and inconsistent rules from the rule base which doesn't affect the performance of the fuzzy logic controller. The FLC based PSS has two input signals as speed deviation and derivative of speed deviation with an appropriate number of linguistic variables. The number of compressed rules in the rule base through the proposed dominant rule algorithm is reduced to a number as low in the number of selected linguistic variables to represent input and output signals. The application of the FLC with compressed rules as a power system stabilizer (CR-FPSS) is investigated by simulation studies on a single-machine infinite-bus system (SMIB). The superior performance of this compressed rule based fuzzy PSS (CR-FPSS) as compared to conventional PSS and proves the better efficiency of this new CR-FPSS. The reduced CPU computational time and storage space as compared to the fuzzy power system stabilizer (FPSS), proves its applicability in control.
\end{abstract}

Keywords: Fuzzy Logic Controller, Rule Base Compression, Compressed Rule FPSS, Single Machine Infinite Bus System, Power System Stabilizer, Dominant Fuzzy Rule Compression

\section{Introduction}

The electric power demand is increasing with time resulting in extension to the power system network \& constraints. These large networks are operated close to their dynamic stability even though it may lead to major system black-outs because of small signal oscillations $(0.1-3 \mathrm{~Hz})$. These can be damped-out by using conventional power system stabilizers (CPSS) because of their flexibility, easy implementation \& of low cost [1, 2]. These CPSSs are the fixed parameter controllers, designed over a nominal operating point to get desired performance at this point as well expect over a wide range of operating conditions and varying system conditions. The CPSSs constitutes a gain amplifier block, lead-lag network and washout block [1]. These CPSS are limited in performance because these may lose effective damping for a wide range of operating conditions within a power system [3].

In 1960 - 70, the classical optimization methods were introduced but not able to converge for non-linear and non-differential engineering problems. Recently, some of the optimization methods such as the particle swarm optimization
(PSO) [4, 5], genetic algorithm (GA) [6, 7], bat algorithm [8] and differential evolution (DE) [9] algorithm, have been applied to complicated and large dimensional power system problems. As a very new optimization method named as Harmony Search Algorithm has been introduced by Geem et al. in 2001 [10], is inspired from the process of the improvisation used by musicians to achieve the harmony. The HS algorithm [11, 12] is a meta-heuristic optimization algorithm which is similar to the PSO [4] and GA [6]. It has been implemented extensively in the fields of engineering optimization [11], in recent years. It became an alternative to other heuristic algorithms like PSO [4], Tabu search [13], simulated annealing $[14,15]$, evolutionary programming [16], rule based bacteria foraging [17] and type-2 fuzzy power system stabilizer $[18,19]$.

In case of adaptive PSS, with poor initialization, the performance during learning phase is not satisfactory. Continuity of the objective function is a prerequisite for gradient algorithm used in such applications [20]. As alternative control theories such as the variable structure, the adaptive and linear optimal control theory has been used to design Power System Stabilizers with improved performance 
in $[20,21]$. Fuzzy logic applications to design of the PSS have emerged as a powerful technique which outperforms the CPSS over a wide range of operating conditions of the power system [22]. The special aspect of Fuzzy PSS is its application to ill-defined systems because of its model-free ability as in [22, 23].

In 1970s [24], the applications of fuzzy control in the engineering field were introduced by design of a pilot boiler-steam engine. The application of systematic rule base as a controller is described in $[25,26]$. The construction of a fuzzy controller is simpler to its tuning and analysis [27, 28]. The major concern of the researchers is to obtain the high interactivity of the rules, completeness and inconsistencies of the fuzzy rules as reported in $[21,29,30]$. The inconsistency and completeness conviction is being used for the study/analysis of the fuzzy controllers. Therefore, a methodology is carried out to analyze the consistency of rule base for FPSS. The inconsistencies of a rule base are determined by equalities of two fuzzy sets. This method is an alternative to the possibility approach proposed in [31, 32].
The refinement in the rule base is carried out by redundancy approach as proposed in [31]. As the redundant rules do not participate/contribute to the performance analysis of the fuzzy controller, therefore, the rule base can be modified by removing these redundant rules. The main considerable aspects of a fuzzy control are performance criteria and cost factors, which affect the size of rule base [31]. The only problem associated with the fuzzy systems is the large number of rules depending upon the number of inputs. The increased number of rules leads to complexity of quality in terms of poor transparency and unclear/vague interpretation of the fuzzy rules, while the quantitative complexity vests in terms of the high number of rules. The variation of a number of rules with the number of linguistic variables for a particular number of inputs is shown in Figure 1 and may be concluded that the number of rule's increases exponentially with the increase of linguistic variables. If the number of inputs is ' $i$ ', if the number of linguistic variables is ' $v$ ', then the number of rules ' $r$ ' is given by $r=v^{i}$. (a): 2-Inputs

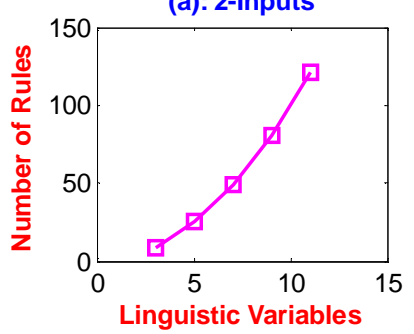

(c): 4-Inputs

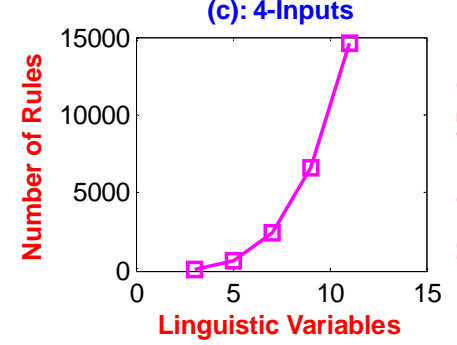

(b): 3-Inputs

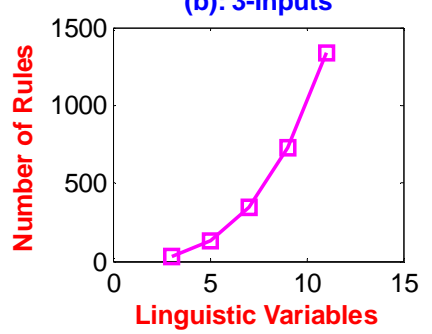

(d): 2,3,4-Inputs

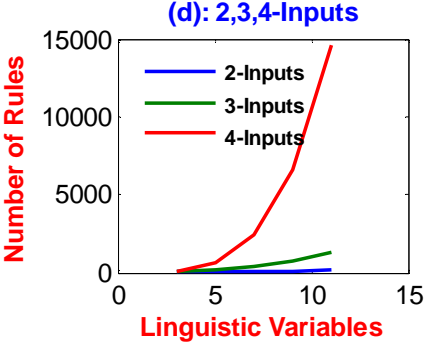

Figure 1. Variation of number of rules for a fuzzy system with two, three and four inputs.

It is well established that fuzzy control is applicable to the systems which are not well defined, complex, possesses uncertainties and cannot be described by mathematical modelling $[12,33]$.The uncertainty is the part of decision making units and is inherent to the systems, where the information is gathered. The reliability of the information-gathering process and the quality of decisions may be seriously affected by these uncertainties. However, it suffers from serious concern to non availability of a standard design procedure, reducibility, optimality and partitioning of the fuzzy rule set. A rule base can be generated by some learning process, identification scheme and more generally from the expert operators can have weakly been contributing rules, redundant rules or inconsistent components of the rules. It is also notable that enlarged rule base suffers from the higher computational time and large storage space. Therefore, an optimal rule base is the requirement of fuzzy systems, thus the present work is an attempt in this direction by using rule compression strategy as suggested in [12, 29, 32, 34, 35].

This paper presents a new fuzzy PSS with compressed rule base for the system data at a nominal operating condition of the power system and applied to different eight operating conditions with fault based disturbance to examine the robustness of it. The performance with this CR-FPSS is compared to conventional PSS and the original FPSS. The special aspect of this method is to partition the input space in a resilient manner [32, 34].

The rest of this paper is organized as follows. In Section 2, the review of the fuzzy system is incorporated. The rule base compression algorithm is presented in section 3 . In section 4, the considered test system for small signal stability improvement using SMIB power system model and dynamics is incorporated. The system is tested with CPSS, with FPSS and with proposed CR-FLPSS. Finally, the observation based 
conclusion is carried out in section 5 .

\section{Operation Stages in Fuzzy Systems}

The pioneer work on fuzzy logic was introduced in 1960's by Prof. Lofti Zadeh [36]. In fuzzy logic, a programmer deals with the natural, "linguistic sets" of states, such as a large negative medium negative, negative, positive, medium positive, large positive, etc. The main parts to the fuzzy logic process are fuzzification (crisp value to fuzzy input), rule evaluation (fuzzy control), and defuzzification (fuzzy output to crisp value) $[37,38]$. The FLC has the following main elements as in Figure 2.

1. Rule-Base: It contains fuzzy logic quantification (a set of If-Then rules) of the expert's linguistic description to the system to get good control.

2. Inference Mechanism: It is also called as "inference engine" or "fuzzy inference" module. It emulates the decision making of expert in interpreting and applying to control the plant.

3. Fuzzification Interface: It converts controller inputs into the fuzzy input, which is used by the inference mechanism to activate and apply rules. The crisp value inputs are converted to fuzzy inputs as the basis of membership functions defined for the fuzzy system.

4. Defuzzification Interface: It converts the output/conclusions of the inference into crisp inputs to the plant.

The purpose of the fuzzy control system is to replace a human expert by a fuzzy rule-based system. The FLC can convert the linguistic control based on expert knowledge into an automatic control strategy. The heart of Fuzzy Systems is a knowledge base, which is formed by the if-then rules of fuzziness.

Fuzzy sets in fuzzy systems are used to map input to output as in Figure 2. The FIS system based on Mamdani as in [24], is considered in this paper. The major stages of mapping involve as fuzzification process, inference process and defuzzification process. The triangular membership functions are used in fuzzification stage $[24,34,39]$. The major parts of an inference system are application, implication and aggregation [34]. The conjunctive process (MIN) is applied in application part, the truncation process on the implication part while a disjunctive process (MAX) is applied in the aggregation part as in Figure 2. There are many defuzzification methods as centroid, bisector, LOM, MOM, SOM but most widely used centroid method is considered in the defuzzification stage [34].

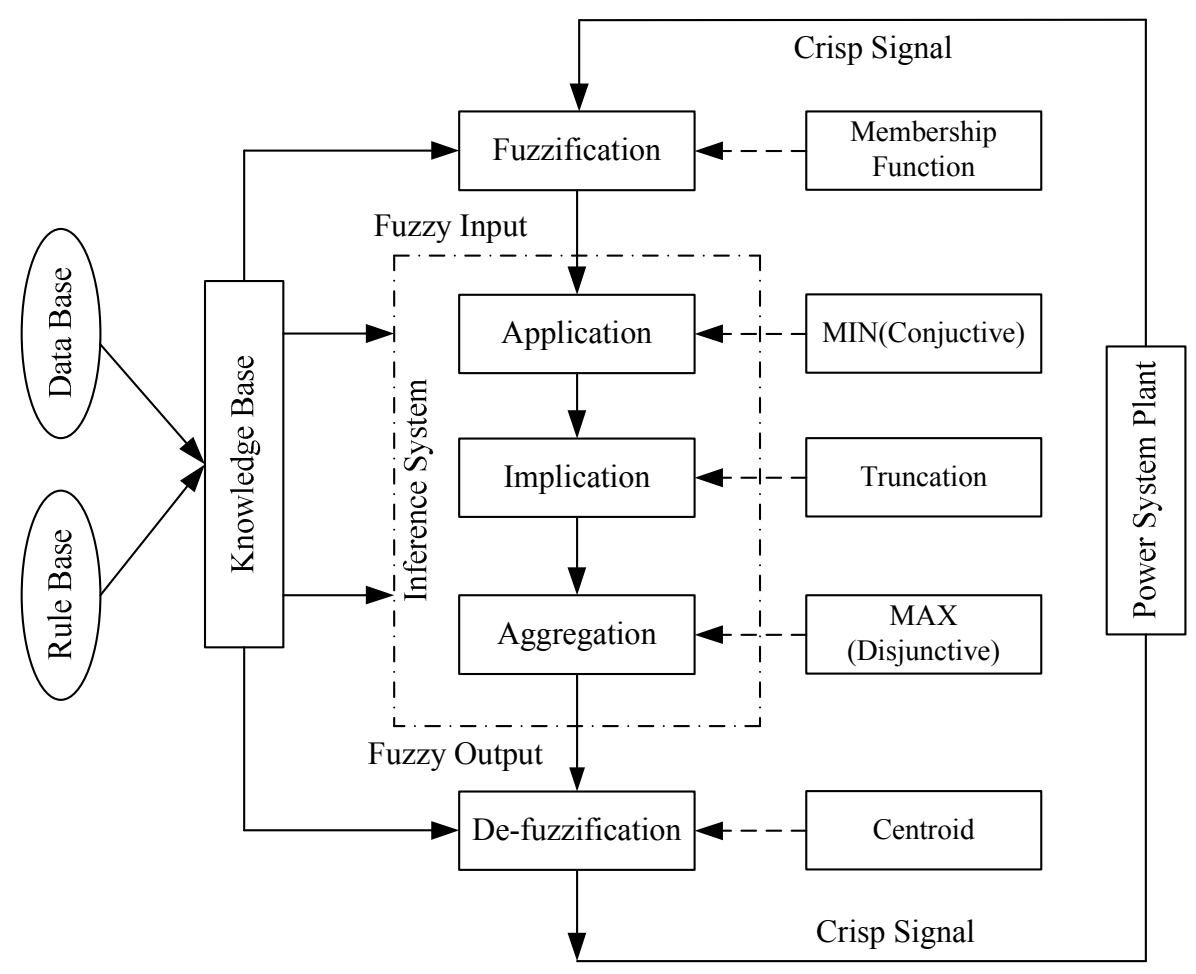

Figure 2. Input to Output mapping in a fuzzy system.

\section{Review on Rule Base Compression Algorithm}

The efforts are made for the rule base reduction in fuzzy control like interpolation algorithm in [32, 40, 41]. In interpolation algorithm, only minimal numbers of necessary rules remain at the rule base. Another approach is boolean used for measuring equivalence and inconsistency in the rule base. This method of rule base reduction with application as a robot manipulator control is proposed by Bezine in [42]. Xiong and Litz [43] have proposed an application of genetic 
algorithm to premise learning methodology for rule base reduction resulting to efficient control of an inverted pendulum.

In this paper, the inconsistency and redundancy as in [31] and dominant rule selection as in $[32,34]$ are used to eliminate highly redundant rules and design of compressed rule base. It is established that the rule's redundancy or non dominant do not contribute to the performance of the controller as FPSS. Therefore, it is necessary to reduce/compress the rule base for fuzzy inference system (FIS) computations in real time. The term redundancy is the measure of the degree to which the input and output fuzzy rules tend to overlap are introduced in $[32,34]$. Therefore, the input and output spaces of any two fuzzy rules closely similar to each other are termed as highly redundant. Thus, the identical rules have redundancy values as 1.0 and totally different rules have a redundancy value as 0 (no value). As in[32], the fuzzy rules in a rule base possess a high degree of redundancy levels may be removed to reduce the size of rule base and maintaining the almost similar performance as with original FIS system.

As the rules having the similar linguistic value of their outputs are called as monotonic rules and are commonly found in fuzzy systems. In the proposed method, monotonic rules are arranged in groups, and the dominant rules are determined for each group. The rules with highest firing strength are called dominant because all other rules from the same group don't have an appreciable impact on the output. The phenomenon is termed as redundancy and would be the basis to rule base compression process. An algorithm for rule base compression can be described as.

Creation of rule base integer table: The rule base integer can be created in two ways: (a) creation by user input, (b) creating from the existing FIS system.

(a) Creation by user input: the user is required to enter information such as the number of inputs and outputs along with the number of linguistic variables for each input and output. The entry for the output values for each possible combination of input linguistic values. According to the entries of input, output and rules, an integer table is created.

(b) Creation from an existing FIS system: in another option, the pre-defined FIS system is loaded and the linguistic variables for inputs and output are assigned by integer numbers. According to the rules within the FIS system, an integer table is created.

1. Input system information: In case of 1(a), the user is required to enter all the information regarding the fuzzy inference system. The information regarding membership function and names for linguistic variables are entered.

2. Creation of the fuzzy rule base with the original rules: In case of 1 (a), according to the entries in step 1and 2, the FIS system is created and saved with innovative details.

3. Rearrange the rules into groups: This step is related to aggregation and is an entirely off-line process. The integer rule table created in step-1 is rearranged in groups and is sorted in ascending order with respect to the chosen output. In a group say output as 2 , then in the second group all rules in terms of integers should be accommodated and similarly for others. If a $7 \times 7$ fuzzy relation matrix (FRM) is created or loaded, afterwards there would be seven groups with outputs as 1, 2, 3, 4, 5, 6 and 7.

4. Find dominant rule and compressed rules: In this step, dominant rue for each group is determined. Since the dominant rules can be determined after the completion of application and fuzzification stages, therefore, the process is only possible on-line. The determination of dominant rules from each group and arranging in a table is called as reduced/compressed integer rule table.

5. Creation of fuzzy rule base with compressed rules: The reduced rule table found in step 5, for an original rule table either by creating or by loading is saved in this step by different names with *.fis as an extension. Let created/loaded FIS system is pss.fis then the reduced should be saved as pssr.fis.

6. Apply fuzzification, inference and defuzzification: This Step is concerned with the evaluation of the file saved in step six. It evaluates the output of a pssr.fis for given inputs like speed as 0.05 , acceleration as 0.005 then the output voltage comes as 0.68 .

7. Generate the solution surface: In this step output-surface is generated for original and reduced FIS system by an applied number of points (generally 30-50) for the crisp input values and the defuzzified output values. It validates the compressed system with respect to the original system.

On application of the algorithm as in $[32,34]$ and following as mentioned above, the monotonic rules remain in the fuzzy rule base. It should be cleared that the number of monotonic rules as the number of linguistic variables or number of groups in the original fuzzy rule base.

It is suggested in $[32,34]$, that the non-monotonic rules should be completely removed from the new rule base. It should also be taken into consideration that the aggregation process to determine the inconsistent rules should always be off-line, while, that of filtering process for determining non-monotonic rules should be carried out on-line. In above algorithm step 1 is carried out off-line while the steps 2-3 are carried out online. The rule with maximal firing strength out of a group is called as a dominant rule. The other rules don't have adequate effect. If a group has more than one dominant rule than random selection can be done to select powerful rule.

The rule base compression is carried out in the MATLAB Fuzzy Logic Toolbox. The algorithm is applied for $7 \times 7$ fuzzy rule matrix with 49 rules. The corresponding rule base is being represented by integer tables in which the linguistic values of inputs and outputs are replaced by integers. The rule-base compression method with the dominant rule method is applied because of its systematic nature and wide applicability. The considered case of 49 rules with speed and acceleration as input and voltage as output being evaluated for rule compression as per algorithm stated above. The procedure is illustrated in the next section. 


\section{Case Study}

\subsection{Test System Description}

The system under consideration consists of the single machine connected to an infinite bus (SMIB) through a tie-line. The infinite bus can be represented by the Thevenin's equivalent of a large interconnected power system. Synchronous generator control components as schematic are shown in Figure 3.

The combinations of a turbine and governor are being used to drive the synchronous generator. The generator is being excited by an external excitation system. The excitation system is controlled by AVR and PSS to control the low-frequency oscillations arise due to lack of damping of the system's mechanical mode. In case of inadequate damping torque, the system may lose stability. Since the high gain, fast acting AVR leads to generate negative damping, therefore, to provide additional damping supplementary excitation control like PSS is developed. The stability to the system may be improved by adding a control loop with the governor, but is limited because of overheating problem and large time constant. Hence, the PSS is the best choice to improve stability $[1,2,44]$.

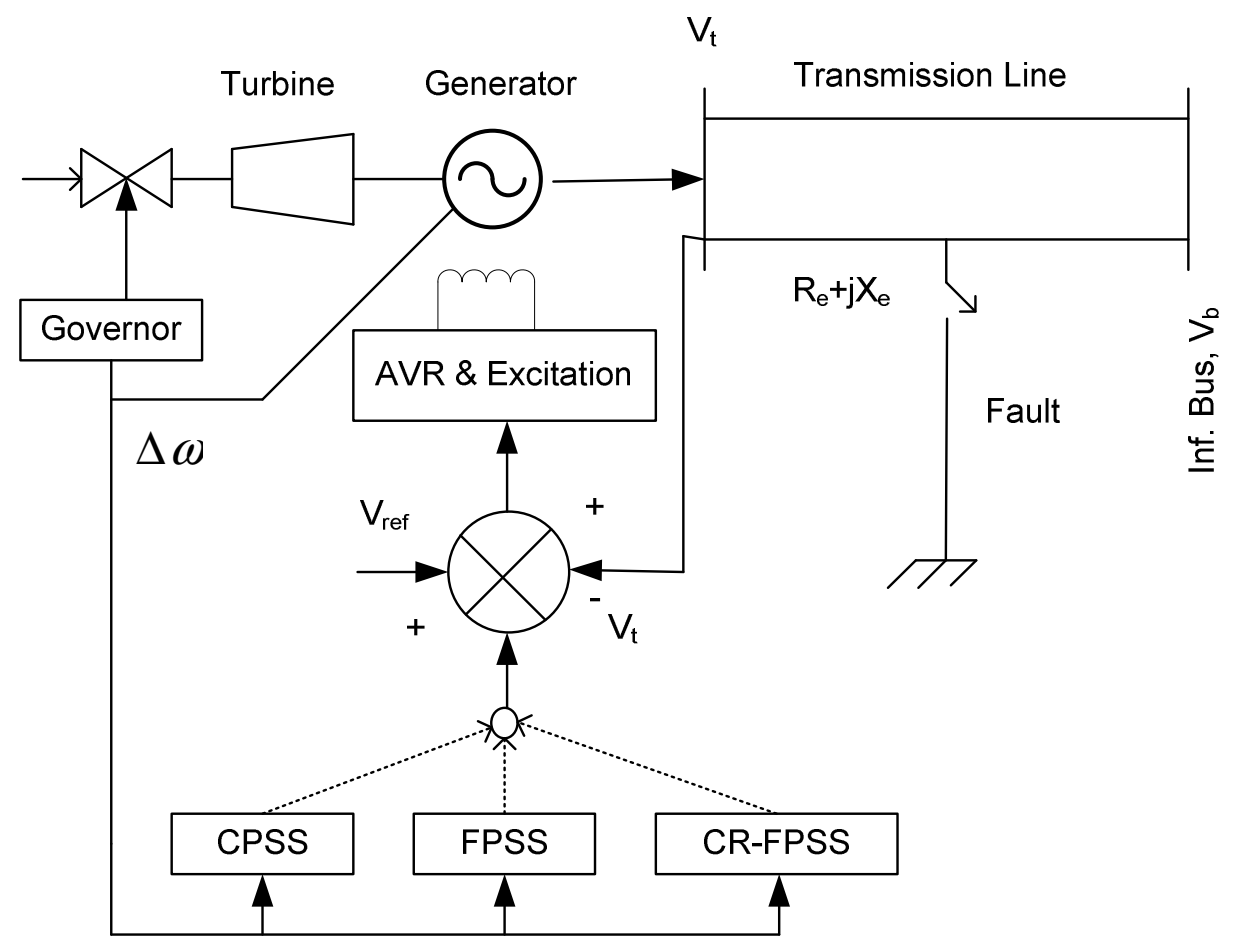

Figure 3. Single Machine Infinite Bus Representation with fault.

Design of conventional PSS is based on a linearized model around a certain operating point. Since the actual power system operates over a wide range of operating conditions and nonlinear characteristics. The tuning of CPSS to cope with most of the operating conditions is very difficult. The change in rotor speed is taken as input to PSS as shown in Figure 4. The structure of PSS is mainly composed by a gain, wash out filter and the phase compensator block $[1,2]$. The gain block is used to ensure the required damping factor in the consideration of practical aspects (operating conditions) of the power system as in $[2,45]$. The washout filter behaves as a high-pass filter; therefore, the PSS only responds to the speed deviation of generator and not responds to the steady-state operation within the system. The criterion to select the washout time constant $T_{\omega}$ is to pass required PSS signals intact $[1,2,46]$. The main concern of the damping issue is the lag compensation in between excitation input and the air gap electrical torque is provided by third block of CPSS, called as the phase compensator block and showed in Figure 4. The output of the PSS is controlled by the limiting block to prevent the over excitation and concerned block is called as limiting block with bounds generally as \pm 0.12 to $\pm 0.15 \mathrm{pu}[1,2,47]$. The transfer function the conventional PSS (CPSS) is given by.

$$
G_{P S S}(s)=K_{p s s} \frac{s T_{\omega}}{1+s T_{\omega}} \frac{1+s T_{1}}{1+s T_{2}}
$$

In the SMIB representation, the dynamic interference between the several machines in the power station is neglected, but it is still an appropriate to various types of studies, especially for identical machines, operating at similar load levels. The linearized model of SMIB was the result of a first serious investigation by DeMello and Concordia in 1969 [48]. This model is used in the study of PSS design and is also adopted illustrating the proposed design method. The static exciter of a single time constant automatic voltage regulator (AVR) is connected to the synchronous generator and is 
represented by.

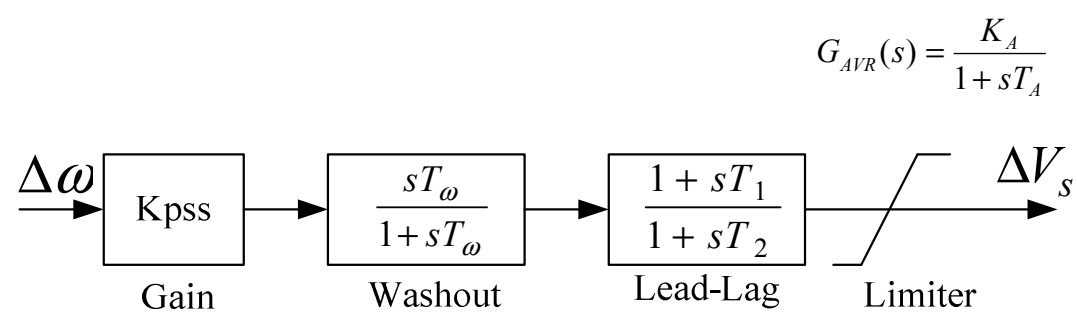

Figure 4. Conventional Power System Stabilizer.

The small perturbation model of the system as in [12], includes the IEEE-type ST1A exciter and the PSS. The linearised differential equations can be written as follows

$$
\begin{gathered}
\Delta \dot{\omega}=-\frac{K_{1}}{2 H} \Delta \delta-\frac{K_{2}}{2 H} \Delta E_{q}^{\prime} \\
\Delta \dot{\delta}=\omega_{0} \Delta \omega \\
\Delta \dot{E}_{q}^{\prime}=-\frac{1}{K_{3} T_{d 0}^{\prime}} \Delta E_{q}^{\prime}-\frac{K_{4}}{T_{d 0}^{\prime}} \Delta \delta+\frac{1}{T_{d 0}^{\prime}} \Delta E_{f d} \\
\Delta E_{f d}=-\frac{K_{A} K_{5}}{T_{A}} \Delta \delta-\frac{K_{A} K_{6}}{T_{A}} E_{q}^{\prime}-\frac{1}{T_{A}} \Delta V_{r e f}
\end{gathered}
$$

By using equation (3)-(6), state space form of the system can be arranged as follows

$$
\begin{gathered}
\dot{x}(t)=A x(t)+B x(t) \\
x(t)=\left[\begin{array}{cccc}
\Delta \omega & \Delta \delta & \Delta E_{q}^{\prime} & \Delta E_{f d}
\end{array}\right]^{T} \\
\left.\begin{array}{cccc}
-\frac{K_{D}}{2 H} & -\frac{K_{1}}{2 H} & -\frac{K_{2}}{2 H} & 0 \\
0 & 0 & 0 & 0 \\
0 & -\frac{K_{4}}{T_{d 0}^{\prime}} & -\frac{1}{K_{3} T_{d 0}^{\prime}} & \frac{1}{T_{d 0}^{\prime}} \\
0 & -\frac{K_{A} K_{5}}{T_{A}} & -\frac{K_{A} K_{6}}{T_{A}} & -\frac{1}{T_{A}}
\end{array}\right] \\
B=\left[\begin{array}{cccc}
0 & 0 & \frac{K_{A}}{T_{A}}
\end{array}\right]
\end{gathered}
$$

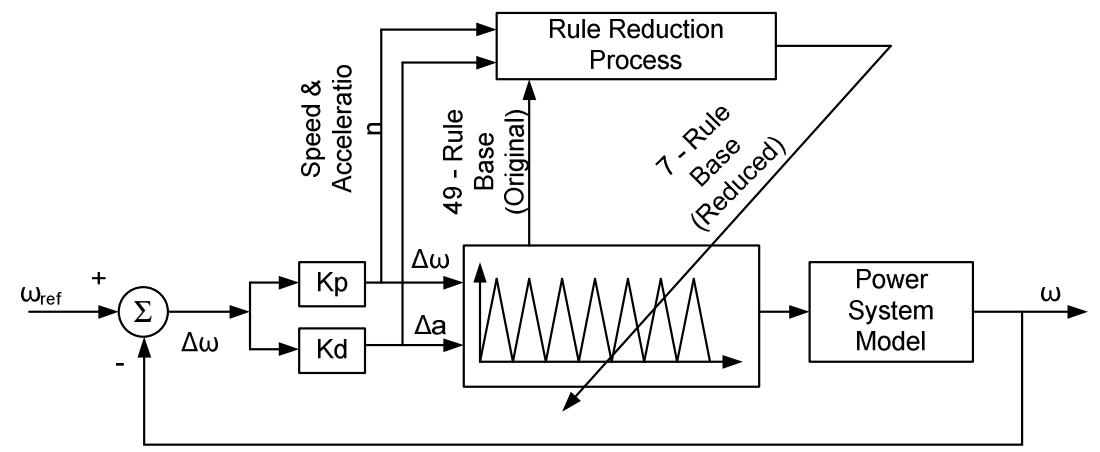

Figure 5. Rule reduction process.
The FLPSS rules are determined by using the proposed rule compression method. Eight operating points of SMIB power system (i.e power system pants as $01-8$ and shown in Table 1). The plants of SMIB power system with FLPSS are simulated in an SIMULINK working environment of MATLAB.

Table 1. SMIB Power System Plants.

\begin{tabular}{llll}
\hline $\begin{array}{l}\text { Power System } \\
\text { Plant }\end{array}$ & $\begin{array}{l}\text { Active Power, } \\
\text { Pg0 }\end{array}$ & $\begin{array}{l}\text { Reactive } \\
\text { Power, Qg0 }\end{array}$ & Reactance, Xe \\
\hline 1 & 0.50 & 0.0251 & 0.2 \\
2 & 0.75 & 0.0566 & 0.2 \\
3 & 1.00 & 0.1010 & 0.2 \\
4 & 1.25 & 0.1588 & 0.2 \\
5 & 0.50 & 0.0505 & 0.4 \\
6 & 0.75 & 0.1152 & 0.4 \\
7 & 1.00 & 0.2087 & 0.4 \\
8 & 1.10 & 0.2550 & 0.4 \\
\hline
\end{tabular}

\subsection{Development of Compressed Rule Table}

The number of Linguistic Variables is taken as 7 to each input. The input signals are taken as Speed Deviation (Error) and rate of change of Speed Deviation (derivative of Error) while the output is taken as Change in Voltage. For simplicity, these three signals are being represented as Speed, Acceleration and Voltage. The seven linguistic variables are defined by LN, MN, N, Z, P, MP, and LP as in Table 2.

Table 2. Representation of Linguistic Variables (7×7 FRM).

\begin{tabular}{llll}
\hline S. No. & Symbol & Linguistic Variables & Integer \\
\hline 1 & LN & Large Negative & 1 \\
2 & MN & Medium Negative & 2 \\
3 & N & Negative & 3 \\
4 & Z & Zero & 4 \\
5 & P & Positive & 5 \\
6 & MP & Medium Positive & 6 \\
7 & LP & Large Positive & 7 \\
\hline
\end{tabular}


Table 3. Input and Output Linguistic Variables.

\begin{tabular}{|c|c|c|c|c|c|c|c|c|}
\hline \multicolumn{9}{|c|}{ Acceleration } \\
\hline \multirow{8}{*}{$\begin{array}{l}\text { Speed } \\
\text { Deviati } \\
\text { on }\end{array}$} & & $\mathrm{LN}$ & $\mathrm{MN}$ & $\mathrm{N}$ & Z & $\mathrm{P}$ & MP & LP \\
\hline & LN & LN & LN & LN & MN & $\mathrm{MN}$ & $\mathrm{N}$ & Z \\
\hline & $\mathrm{MN}$ & LN & LN & $\mathrm{MN}$ & MN & $\mathrm{N}$ & Z & $\mathrm{P}$ \\
\hline & $\mathrm{N}$ & LN & MN & LN & $\mathrm{N}$ & Z & $\mathrm{P}$ & MP \\
\hline & Z & MN & MN & $\mathrm{N}$ & Z & $\mathrm{P}$ & MP & MP \\
\hline & $\mathrm{P}$ & $\mathrm{MN}$ & $\mathrm{N}$ & Z & $\mathrm{P}$ & LP & MP & LP \\
\hline & MP & $\mathrm{N}$ & Z & $\mathrm{P}$ & MP & MP & LP & LP \\
\hline & LP & Z & $\mathrm{P}$ & MP & MP & LP & LP & LP \\
\hline
\end{tabular}

As per the Table 3, the number of output rules is 49 and the table is called as fuzzy relation matrix (FRM) or simply rules table. As per step-5, the dominant rules (only in online process) for selected inputs, i.e. speed as 0.008929 pu and accelerations as $0.08929 \mathrm{pu}$ are determined from the groups as in Table 4. The procedure of speed and acceleration is determined as in Figure 5. The rules are written in the form of IF-THEN rules. The rule table for the original FIS system is arranged by an integer as in step-3 and is shown Table 5(a). The same integer table is being re-arranged into a group rule table and is represented in Table 5(b). The number of groups is equal in the number of linguistic variables. Generally, one dominant rule is selected from each group and resulting in the compressed rule number as 7 in Table 6 . A case may exist that a particular group is having more than one powerful rule then a single rule should be selected on a random basis. As stated above, the reduced rule table for $7 \times 7$ FRM in Table 6 can be written as the linguistic variables as in Table 7 . The dominant linguistic variables enlisted as a result of the online process and resulting rules as in Table 7 is shown by bold and italic in Table 3.

Table 4. Speed and Acceleration for creation of compressed rule base for plant 1 to 8 .

\begin{tabular}{lll}
\hline Plant & Speed & Acceleration \\
\hline Plant-1 & 0.004464 & 0.04464 \\
Plant-2 & 0.006696 & 0.06696 \\
Plant-3 & 0.008929 & 0.08929 \\
Plant-4 & 0.01116 & 0.1116 \\
Plant-5 & 0.004465 & 0.04465 \\
Plant-6 & 0.006697 & 0.06697 \\
Plant-7 & 0.008929 & 0.08929 \\
Plant-8 & 0.009821 & 0.09821 \\
\hline
\end{tabular}

The representation of the bold-italic in Table 3 is same in terms of output linguistic variable as in Table 6 (in integer form) and Table 7 (in LV form). It is the rule base saved with *.fis extension and used for the performance analysis of FPSS using reduced rule FIS. To look into the validation of the primary and decreased rule table, the output surface is generated and the similarity of the output surfaces proves the mimics operation of the reduced rule system to its first counterpart.

\subsection{Test System Response Analysis}

The system considered in the PSS design using a reduced number of rule base of $7 \times 7$ FRM is applied to plants $1-8$ as in Table 1. In this case, the plants being tested with Fuzzy PSS (reduced rule base) and compared to the system response with
CPSS and with FPSS; presented in Figure 6 to 9. In all responses, the system under different operating conditions is stable with CR-FPSS. The computation time taken by CPU, with CPSS, FPSS and proposed CR-FPSS is recorded in Table 8; resulting least with CR-FPSS as compared to FPSS for all plant conditions (lightly loaded to heavy loaded). Here, it should be noted that the computational time in case of CPSS is least in comparison to FPSS and CR-FPSS, but is not able to stabilize on heavy loading conditions like plant-8, shown in Figure 9(b). For further clarification of the system response, the generator speed, control voltage, terminal voltage, air-gap electric torque, angle and excitation voltages are shown in Figure 10(a), 10(b), 11(a), 11(b), 12(a) and 12(b), respectively for nominal operating conditions of the SMIB power system as in plant 3 .

Table 5. Integer Table for Original PSS rule base.

\begin{tabular}{|c|c|c|c|c|c|c|c|}
\hline \multicolumn{4}{|c|}{ (a) Complete integer Rule table } & \multicolumn{4}{|c|}{ (b) Grouped integer Rule table } \\
\hline Rule & Speed & Acc. & Vol. & Rule & Speed & Acc. & Vol. \\
\hline 1 & 1 & 1 & 1 & 1 & 1 & 1 & 1 \\
\hline 2 & 1 & 2 & 1 & 2 & 1 & 2 & 1 \\
\hline 3 & 1 & 3 & 1 & 3 & 1 & 3 & 1 \\
\hline 4 & 1 & 4 & 2 & 4 & 2 & 1 & 1 \\
\hline 5 & 1 & 5 & 2 & 5 & 2 & 2 & 1 \\
\hline 6 & 1 & 6 & 3 & 6 & 3 & 1 & 1 \\
\hline 7 & 1 & 7 & 4 & 7 & 3 & 3 & 1 \\
\hline 8 & 2 & 1 & 1 & 8 & 1 & 4 & 2 \\
\hline 9 & 2 & 2 & 1 & 9 & 1 & 5 & 2 \\
\hline 10 & 2 & 3 & 2 & 10 & 2 & 3 & 2 \\
\hline 11 & 2 & 4 & 2 & 11 & 2 & 4 & 2 \\
\hline 12 & 2 & 5 & 3 & 12 & 3 & 2 & 2 \\
\hline 13 & 2 & 6 & 4 & 13 & 4 & 1 & 2 \\
\hline 14 & 2 & 7 & 5 & 14 & 4 & 2 & 2 \\
\hline 15 & 3 & 1 & 1 & 15 & 5 & 1 & 2 \\
\hline 16 & 3 & 2 & 2 & 16 & 1 & 6 & 3 \\
\hline 17 & 3 & 3 & 1 & 17 & 2 & 5 & 3 \\
\hline 18 & 3 & 4 & 3 & 18 & 3 & 4 & 3 \\
\hline 19 & 3 & 5 & 4 & 19 & 4 & 3 & 3 \\
\hline 20 & 3 & 6 & 5 & 20 & 5 & 2 & 3 \\
\hline 21 & 3 & 7 & 6 & 21 & 6 & 1 & 3 \\
\hline 22 & 4 & 1 & 2 & 22 & 1 & 7 & 4 \\
\hline 23 & 4 & 2 & 2 & 23 & 2 & 6 & 4 \\
\hline 24 & 4 & 3 & 3 & 24 & 3 & 5 & 4 \\
\hline 25 & 4 & 4 & 4 & 25 & 4 & 4 & 4 \\
\hline 26 & 4 & 5 & 5 & 26 & 5 & 3 & 4 \\
\hline 27 & 4 & 6 & 6 & 27 & 6 & 2 & 4 \\
\hline 28 & 4 & 7 & 6 & 28 & 7 & 1 & 4 \\
\hline 29 & 5 & 1 & 2 & 29 & 2 & 7 & 5 \\
\hline 30 & 5 & 2 & 3 & 30 & 3 & 6 & 5 \\
\hline 31 & 5 & 3 & 4 & 31 & 4 & 5 & 5 \\
\hline 32 & 5 & 4 & 5 & 32 & 5 & 4 & 5 \\
\hline 33 & 5 & 5 & 7 & 33 & 6 & 3 & 5 \\
\hline
\end{tabular}




\begin{tabular}{llllllll}
\hline \multicolumn{3}{l}{ (a) Complete integer Rule table } & \multicolumn{3}{c}{ (b) Grouped integer Rule table } \\
\hline Rule & Speed & Acc. & Vol. & Rule & Speed & Acc. & Vol. \\
\hline 34 & 5 & 6 & 6 & 34 & 7 & 2 & 5 \\
35 & 5 & 7 & 7 & 35 & 3 & 7 & 6 \\
36 & 6 & 1 & 3 & 36 & 4 & 6 & 6 \\
37 & 6 & 2 & 4 & 37 & 4 & 7 & 6 \\
38 & 6 & 3 & 5 & 38 & 5 & 6 & 6 \\
39 & 6 & 4 & 6 & 39 & 6 & 4 & 6 \\
40 & 6 & 5 & 6 & 40 & 6 & 5 & 6 \\
41 & 6 & 6 & 7 & 41 & 7 & 3 & 6 \\
42 & 6 & 7 & 7 & 42 & 7 & 4 & 6 \\
43 & 7 & 1 & 4 & 43 & 5 & 5 & 7 \\
44 & 7 & 2 & 5 & 44 & 5 & 7 & 7 \\
45 & 7 & 3 & 6 & 45 & 6 & 6 & 7 \\
46 & 7 & 4 & 6 & 46 & 6 & 7 & 7 \\
47 & 7 & 5 & 7 & 47 & 7 & 5 & 7 \\
48 & 7 & 6 & 7 & 48 & 7 & 6 & 7 \\
49 & 7 & 7 & 7 & 49 & 7 & 7 & 7 \\
\hline
\end{tabular}

Table 6. Reduced Rule Integer Table for PSS.

\begin{tabular}{lll}
\hline \multicolumn{3}{l}{ Initial Parameters of FPSS for Compressed Rules } \\
\hline Speed & Acceleration & Voltage \\
\hline 1 & 1 & 1 \\
1 & 4 & 2 \\
1 & 6 & 3 \\
4 & 4 & 4 \\
5 & 4 & 5 \\
3 & 7 & 6 \\
5 & 5 & 7 \\
\hline
\end{tabular}

Table 7. Reduced Rule Table in LV form for PSS.

\begin{tabular}{ll}
\hline Rule No. & Linguistic Rules \\
\hline 1 & If (speed is LN) and (acceleration is LN) then (voltage is LN) \\
2 & If (speed is LN) and (acceleration is Z) then (voltage is MN) \\
3 & If (speed is LN) and (acceleration is MP) then (voltage is N) \\
4 & If (speed is Z) and (acceleration is Z) then (voltage is Z) \\
5 & If (speed is P) and (acceleration is Z) then (voltage is P) ) \\
6 & If (speed is N) and (acceleration is LP) then (voltage is MP) \\
7 & If (speed is P) and (acceleration is P) then (voltage is LP) \\
\hline
\end{tabular}

Table 8. CPU Computational Time.

\begin{tabular}{llll}
\hline $\begin{array}{l}\text { Power System } \\
\text { Model }\end{array}$ & $\begin{array}{l}\text { CPU time for } \\
\text { CPSS }\end{array}$ & $\begin{array}{l}\text { CPU time for } \\
\text { Fuzzy PSS }\end{array}$ & $\begin{array}{l}\text { CPU time for } \\
\text { CFPSS (Prop.) }\end{array}$ \\
\hline Plant-1 & 0.7958 & 130.1118 & 23.0398 \\
Plant-2 & 0.7256 & 127.3183 & 22.1662 \\
Plant-3 & 0.7159 & 128.7899 & 22.3365 \\
Plant-4 & 0.7486 & 155.4953 & 24.8997 \\
Plant-5 & 0.5609 & 137.367 & 21.6231 \\
Plant-6 & 0.6083 & 140.6716 & 22.2323 \\
Plant-7 & 0.73 & 138.4199 & 25.0446 \\
Plant-8 & 1.3983 & 141.1053 & 26.4898 \\
\hline
\end{tabular}

(a) Plant-1

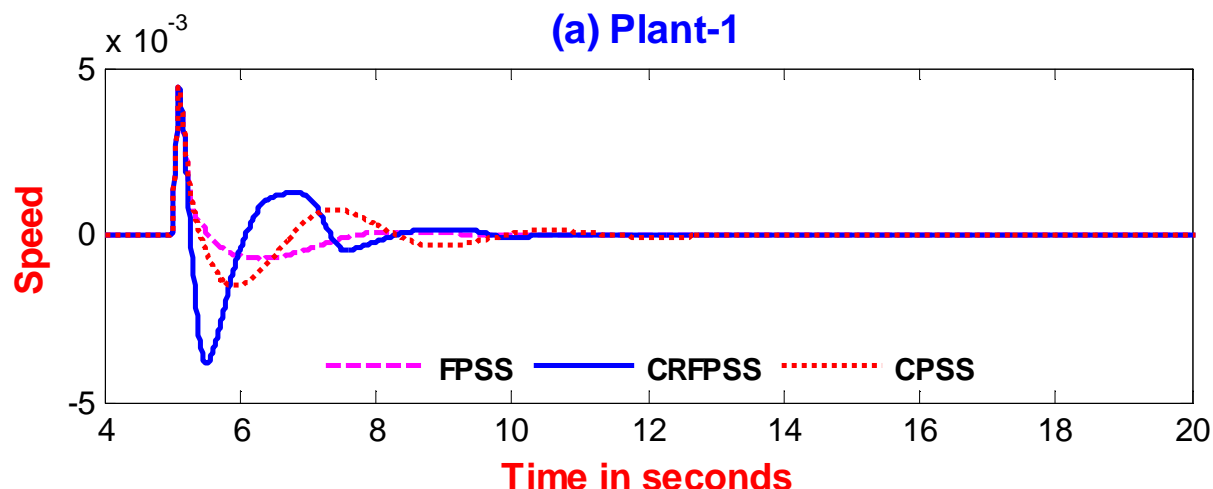

(b) Plant-2

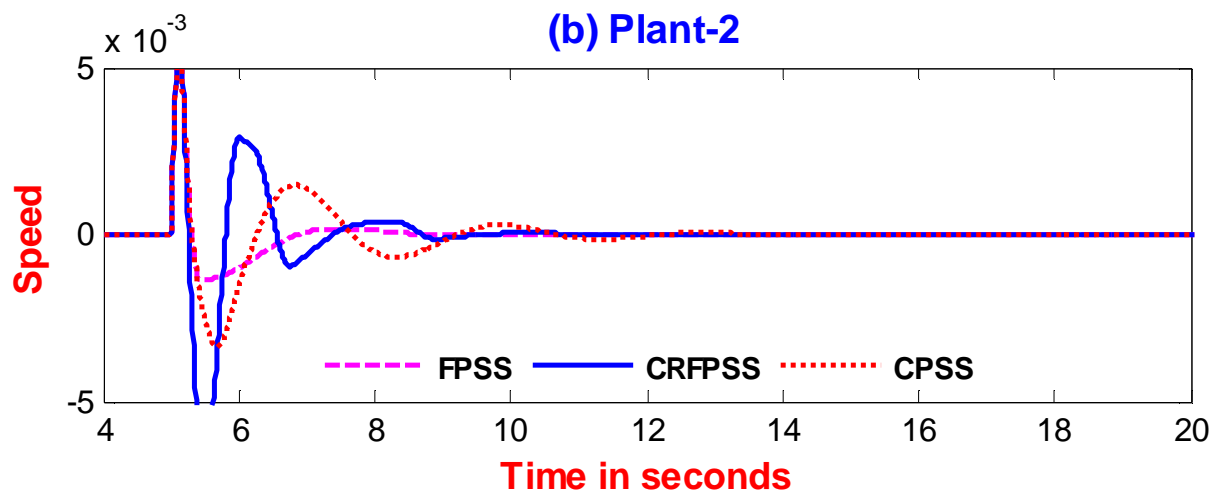

Figure 6. Speed response with CRFPSS, FPSS and CPSS for (a) Plant-1, and (b) Plant-2. 
(a) Plant-3

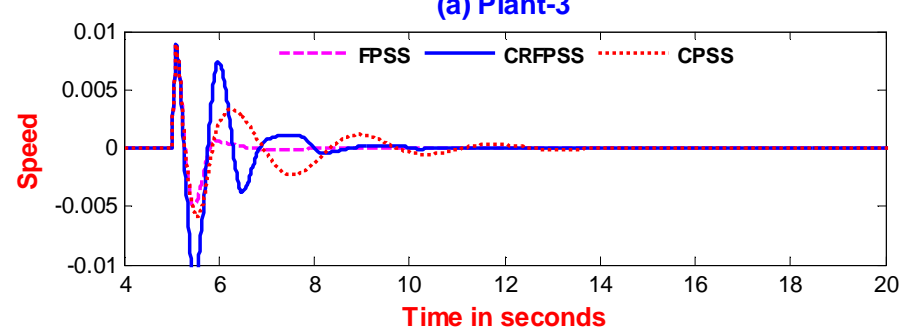

(b) Plant-4

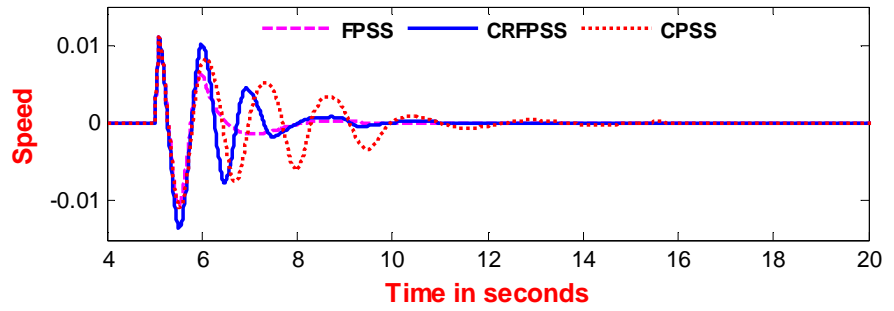

Figure 7. Speed response with CRFPSS, FPSS and CPSS for (a) Plant-3, and (b) Plant-4.
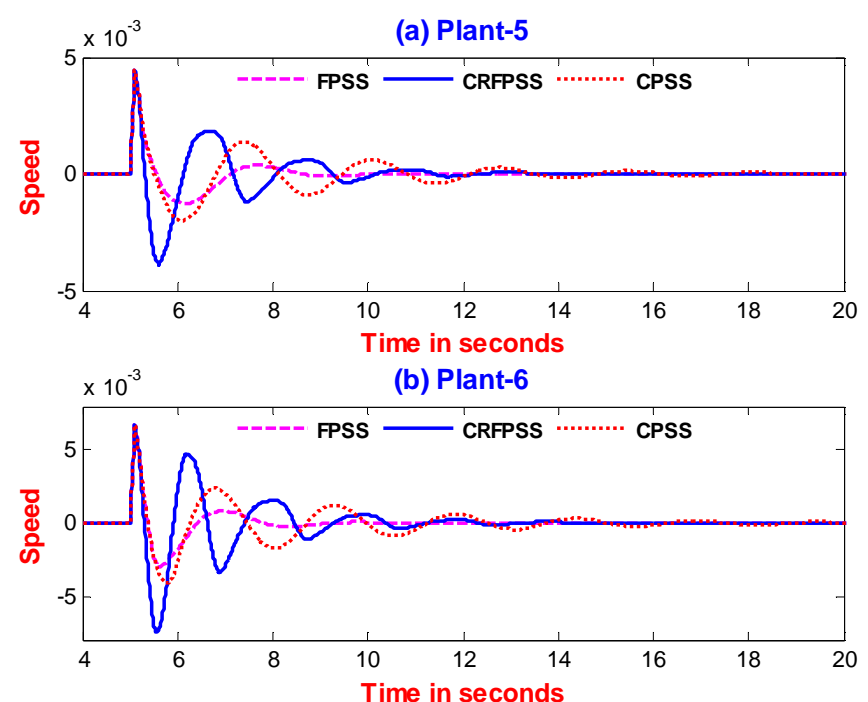

Figure 8. Speed response with CRFPSS, FPSS and CPSS for (a) Plant-5, and (b) Plant-6

(a) Plant-7

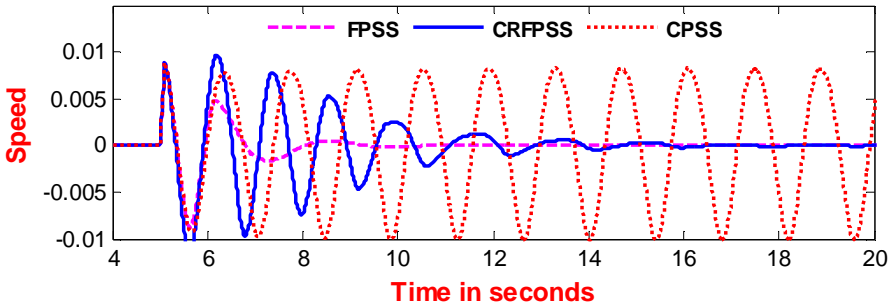

(b) Plant-8

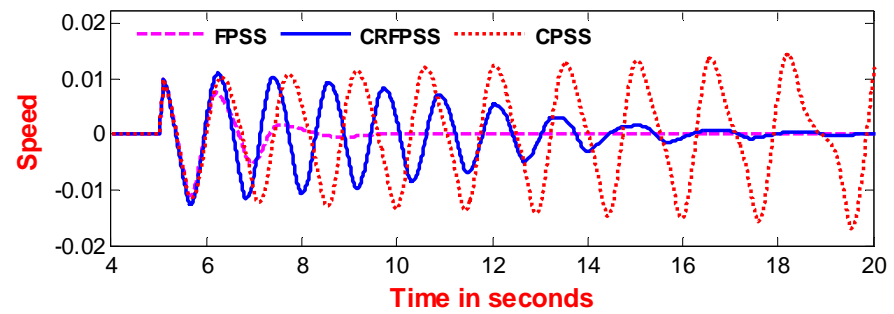

Figure 9. Speed response with CRFPSS, FPSS and CPSS for (a) Plant-7, and (b) Plant-8. 
(a) Speed for NOP

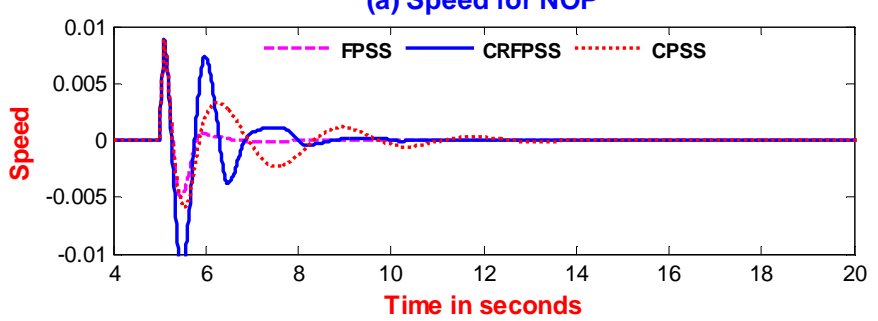

(b) Control Voltage for NOP

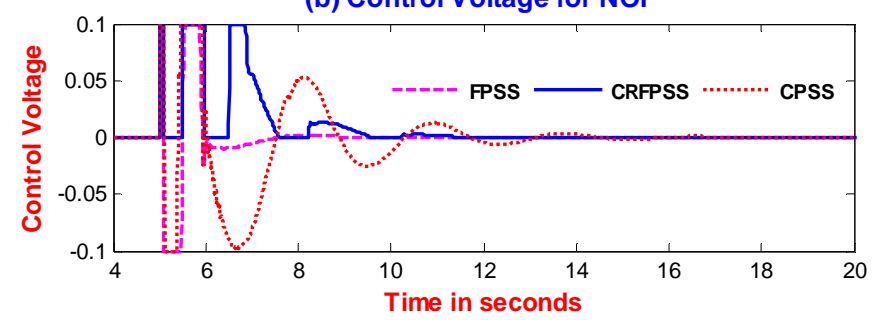

Figure 10. Response with CRFPSS, FPSS and CPSS at nominal operating point for (a) Speed, and (b) Control Voltage.

(a) Terminal Voltage for NOP

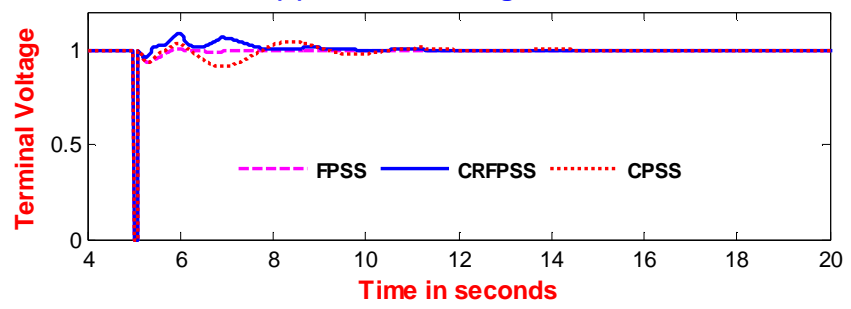

(b) Electric Torque for NOP

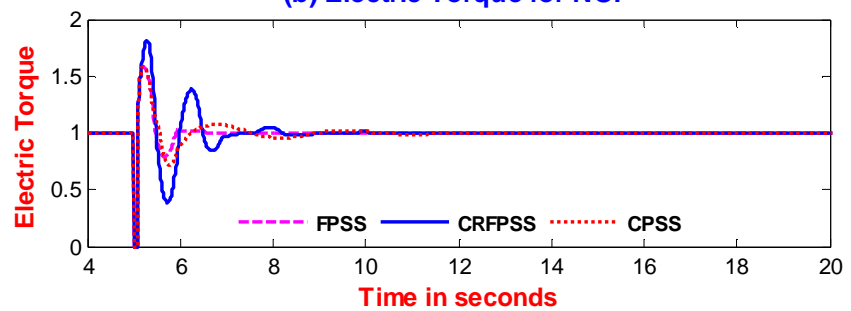

Figure 11. Response with CRFPSS, FPSS and CPSS at nominal operating point for (a) Terminal Voltage, and (b) Electric air-gap torque.

(a) Angle for NOP

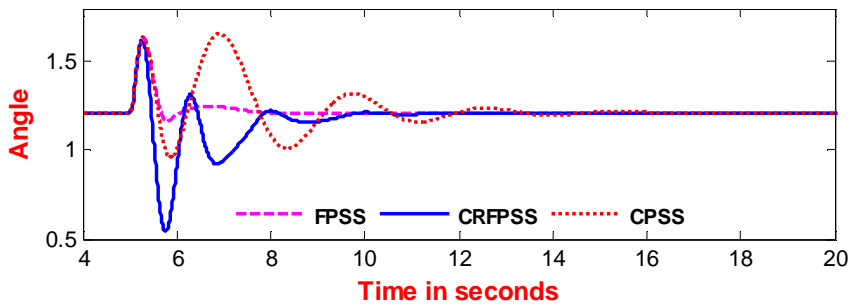

(b) Eq for NOP

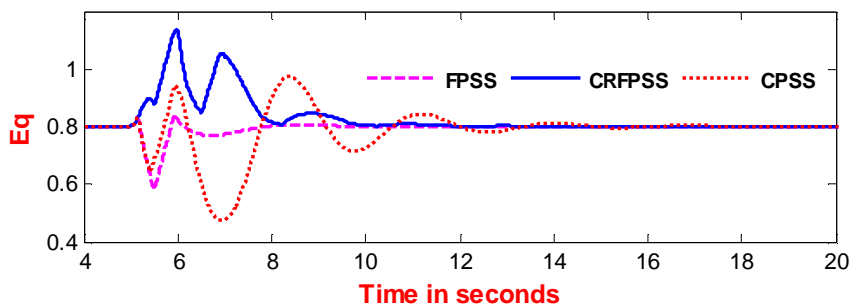

Figure 12. Response with CRFPSS, FPSS and CPSS at nominal operating point for (a) Angle, and (b) Excitation Input (Eq'). 


\section{Conclusion}

A novel rule reduction algorithm is applied to design a fuzzy logic based power system stabilizer. The reduced rule based FLC is applied to a single-machine infinite-bus system as the power system stabilizer for small signal stability improvement.

The proposed method (dominant rule reduction) based designed CR-FPSS is applied by considering $7 \times 7$ FRM with 49 rules, which is compressed to 7-rules. The application of the reduced tables of FIS system can stabilize the SMIB system for wide operating conditions (as plant 1-8) with decreased settling time as compared to CPSS, while as reduced computation time and memory storage as compared to FPSS. The superior performance of this compressed rule based fuzzy PSS (CR-FPSS) as compared to conventional PSS and proves the better efficiency of this new CR-FPSS.

This method is applicable to the Mamdani type of fuzzy systems and pertinent irrespective of the number of inputs, outputs, linguistic values, membership functions and concerned rules. The dominant rule compression algorithm manages the complexity in the fuzzy systems. In this paper, the algorithm is applied only for a single cycle from original FIS system creation/loading of the reduced FIS system. It can be applied to another number of cycles, but the complexity would be increased. The complexity associated to an increase in cycles is a further scope of research.

\section{References}

[1] P. Kundur, M. Klein, G. J. Rogers, and M. S. Zywno, "Application of power system stabilizers for enhancement of overall system stability," IEEE Transactions on Power Systems, vol. 4, pp. 614-626, 1989. doi:10.1109/59.193836

[2] E. V. Larsen and D. A. Swann, "Applying Power System Stabilizers Part I: General Concepts," IEEE Transactions on Power Apparatus and Systems, vol. PAS-100, pp. 3017-3024, 1981. doi:10.1109/tpas.1981.316355

[3] M. Nambu and Y. Ohsawa, "Development of an advanced power system stabilizer using a strict linearization approach," IEEE Transactions on Power Systems, vol. 11, pp. 813-818, 1996. doi:10.1109/59.496159

[4] S. M. Abd-Elazim and E. S. Ali, "A hybrid Particle Swarm Optimization and Bacterial Foraging for optimal Power System Stabilizers design," International Journal of Electrical Power \& Energy Systems, vol. 46, pp. 334-341, 2013. doi:http://dx.doi.org/10.1016/j.ijepes.2012.10.047

[5] H. M. Soliman, E. H. E. Bayoumi, and M. F. Hassan, "PSO-based Power System Stabilizer for Minimal Overshoot and Control Constraints," Journal of Electrical Engineering, vol. 59, pp. 153-159, 2008 . doi:http://iris.elf.stuba.sk/JEEEC/data/pdf/3_108-6

[6] H. Alkhatib and J. Duveau, "Dynamic genetic algorithms for robust design of multimachine power system stabilizers," International Journal of Electrical Power \& Energy Systems, vol. 45, pp. 242-251, 2013. doi:10.1016/j.ijepes.2012.08.080
[7] S. Panda, "Multi-Objective Non-Dominated shorting Genetic Algorithm-II for Excitation and TCSC-based Controller Design," Journal of Electrical Enginnering, vol. 60, pp. 86-93, 2009. doi:http://iris.elf.stuba.sk/JEEEC/data/pdf/2_109-6

[8] D. K. Sambariya and R. Prasad, "Robust tuning of power system stabilizer for small signal stability enhancement using metaheuristic bat algorithm," International Journal of Electrical Power \& Energy Systems, vol. 61, pp. 229-238, 2014. doi: http://dx.doi.org/10.1016/j.ijepes.2014.03.050

[9] S. Panda, "Robust coordinated design of multiple and multi-type damping controller using differential evolution algorithm," International Journal of Electrical Power \& Energy Systems, vol. 33, pp. 1018-1030, 2011. doi:10.1016/j.ijepes.2011.01.019

[10] Zong Woo Geem, Joong Hoon Kim, and G. V. Loganathan, "A New Heuristic Optimization Algorithm: Harmony Search," Simulation, vol. 76, pp. 60-68, February 1, 20012001. doi:10.1177/003754970107600201

[11] Z. W. Geem, "Harmony Search Applications in Industry," in Studies in Fuzziness and Soft Computing, Volume 226, Soft Computing Applications in Industry. vol. 226, B. Prasad, Ed., ed: Springer-Verlag Berlin Heidelberg, 2008, pp. 117-134. doi:10.1007/978-3-540-77465-5_6

[12] D. K. Sambariya and R. Prasad, "Design of Harmony Search Algorithm based tuned Fuzzy logic Power System Stabilizer," International Review of Electrical Engineering (IREE), vol. 8, pp. 1594-1607, October 2013 2013. doi: http://dx.doi.org/10.15866/iree.v8i5.2117

[13] Y. L. Abdel-Magid, M. A. Abido, and A. H. Mantawy, "Robust tuning of power system stabilizers in multimachine power systems," in IEEE Power Engineering Society Winter Meeting, 2000, p. 1425 vol.2. doi:10.1109/pesw.2000.850180

[14] M. A. Abido, "Robust design of multimachine power system stabilizers using simulated annealing," IEEE Transactions on Energy Conversion, vol. 15, pp. 297-304, 2000. doi:10.1109/60.875496

[15] M. A. Abido, "Simulated annealing based approach to PSS and FACTS based stabilizer tuning," International Journal of Electrical Power \& Energy Systems, vol. 22, pp. 247-258, 2000. doi:10.1016/s0142-0615(99)00055-1

[16] M. A. Abido and Y. L. Abdel-Magid, "Optimal Design of Power System Stabilizers Using Evolutionary Programming," IEEE Transactions on Energy Conversion, vol. 17, pp. 429-436, 2002. doi:10.1109/mper.2002.4312476

[17] S. Mishra, M. Tripathy, and J. Nanda, "Multi-machine power system stabilizer design by rule based bacteria foraging," Electric Power Systems Research, vol. 77, pp. 1595-1607, 2007. doi:10.1016/j.epsr.2006.11.006

[18] D. K. Sambariya and R. Prasad, "Evaluation of interval type-2 fuzzy membership function \& robust design of power system stabilizer for SMIB power system," Sylwan Journal, vol. 158, pp. 289-307, April $2014 \quad 2014$. doi:http://sylwan.ibles.org/archive.php? $\mathrm{v}=158 \& \mathrm{i}=5$

[19] D. K. Sambariya and R. Prasad, "Power System Stabilizer design for Multimachine Power System using Interval Type-2 Fuzzy Logic Controller," International Review of Electrical Engineering (IREE), vol. 8, pp. 1556-1565, October 20132013. doi: http://dx.doi.org/10.15866/iree.v8i5.2113 
[20] P. S. Bhati and R. Gupta, "Robust fuzzy logic power system stabilizer based on evolution and learning," International Journal of Electrical Power \& Energy Systems, vol. 53, pp. 357-366, doi:http://dx.doi.org/10.1016/j.ijepes.2013.05.014

[21] T. Hussein, M. S. Saad, A. L. Elshafei, and A. Bahgat, "Robust adaptive fuzzy logic power system stabilizer," Expert Systems with Applications, vol. 36, pp. 12104-12112, 2009. doi:10.1016/j.eswa.2009.04.013

[22] K. A. El-Metwally and O. P. Malik, "Fuzzy logic power system stabiliser," IEE Proceedings-Generation, Transmission and Distribution, vol. 142, pp. 277-281, 1995. doi:10.1049/ip-gtd:19951748

[23] K. A. El-Metwally and O. P. Malik, "Application of fuzzy logic stabilisers in a multimachine power system environment," IEE Proceedings-Generation, Transmission and Distribution, vol. 143, pp. 263-268, 1996. doi:10.1049/ip-gtd:19960193

[24] E. H. Mamdani and S. Assilian, "An experiment in linguistic synthesis with a fuzzy logic controller," International Journal of Man-Machine Studies, vol. 7, pp. 1-13, 1975. doi:http://dx.doi.org/10.1016/S0020-7373(75)80002-2

[25] N. Hosseinzadeh and A. Kalam, "A rule-based fuzzy power system stabilizer tuned by a neural network," IEEE Transactions on Energy Conversion, vol. 14, pp. 773-779, 1999 doi: $10.1109 / 60.790950$

[26] D. K. Sambariya, R. Gupta, and A. K. Sharma, "Fuzzy Applications to Single Machine Power System Stabilizers," Journal of Theoretical and Applied Information Technology, vol. 5, pp. 317-324, 2009 . doi:http://www.jatit.org/volumes/research-papers/Vol5No3/9V ol5No3.pdf

[27] T. Hiyama, "Application of neural network to real time tuning of fuzzy logic PSS," in Neural Networks to Power Systems, 1993. ANNPS '93., Proceedings of the Second International Forum on Applications of, 1993, pp. 421-426. doi:10.1109/ann.1993.264311

[28] R. Gupta, D. K. Sambariya, and R. Gunjan, "Fuzzy Logic based Robust Power System Stabilizer for Multi-Machine Power System," in IEEE International Conference on Industrial Technology, ICIT 2006., 2006, pp. 1037-1042. doi:10.1109/icit.2006.372299

[29] T. Hussein, M. S. Saad, A. L. Elshafei, and A. Bahgat, "Damping inter-area modes of oscillation using an adaptive fuzzy power system stabilizer," Electric Power Systems Research, vol. 80, pp. 1428-1436, 2010. doi:10.1016/j.epsr.2010.06.004

[30] T. Hussein, A. L. Elshafei, and A. Bahgat, "Design of a hierarchical fuzzy logic PSS for a multi-machine power system," in Mediterranean Conference on Control \& Automation (MED '07') 2007, pp. 1-6. doi:10.1109/med.2007.4433681

[31] M. K. Ciliz, "An advanced tuning methodology for fuzzy control: with application to a vacuum cleaner," in Control Applications, 2003. CCA 2003. Proceedings of 2003 IEEE Conference on, 2003, pp. 257-262 vol.1. doi:10.1109/cca.2003.1223320

[32] A. Gegov, "Complexity Management in Fuzzy Systems," Springer, Berlin, 2007.
[33] L. Wang, R. Yang, P. M. Pardalos, L. Qian, and M. Fei, "An adaptive fuzzy controller based on harmony search and its application to power plant control," International Journal of Electrical Power \& Energy Systems, vol. 53, pp. 272-278, 2013. doi:http://dx.doi.org/10.1016/j.ijepes.2013.05.015

[34] A. Gegov and N. Gobalakrishnan, "Advanced Inference in Fuzzy Systems by Rule Base Compression," Mathware \& Soft Computing, vol. 14, pp. 201-216, 2007.

[35] D. K. Sambariya and R. Gupta, "Fuzzy Applications in a Multi-Machine Power System Stabilizer," Journal of Electrical Engineering \& Technology, vol. 5, pp. 503-510, 2010. doi:http://www.jeet.or.kr/ltkpsweb/pub/pubfpfile.aspx?ppseq= 100

[36] L. A. Zadeh, "Fuzzy Sets," Information Control, vol. 8, pp. 338-353, 1965.

[37] D. Flynn, B. W. Hogg, E. Swidenbank, and K. J. Zachariah, "Expert control of a self-tuning automatic voltage regulator," Control Engineering Practice, vol. 3, pp. 1571-1579, 1995. doi:10.1016/0967-0661(95)00167-s

[38] K. J. Åström and B. Wittenmark, "On self tuning regulators," $\begin{array}{lllll}\text { Automatica, } & \text { vol. } \quad 9, \quad \text { pp. } & 185-199, & 1973 .\end{array}$ doi:10.1016/0005-1098(73)90073-3

[39] J. Dombi, "Membership function as an evaluation," Fuzzy Sets and Systems, vol. 35, pp. 1-21, 1990. doi:10.1016/0165-0114(90)90014-w

[40] Z. Q. Wu, M. Masaharu, and Y. Shi, "An improvement to Kóczy and Hirota's interpolative reasoning in sparse fuzzy rule bases," International Journal of Approximate Reasoning, vol. 15, pp. 185-201, 1996 doi:http://dx.doi.org/10.1016/S0888-613X(96)00054-0

[41] L. Kóczy and K. Hirota, "Interpolative reasoning with insufficient evidence in sparse fuzzy rule bases," Information Sciences, vol. 71, pp. 169-201, 1993. doi:http://dx.doi.org/10.1016/0020-0255(93)90070-3

[42] H. Bezine, N. Derbel, and A. M. Alimi, "Fuzzy control of robot manipulators: - some issues on design and rule base size reduction," Engineering Applications of Artificial Intelligence, vol. 15, pp. 401-416, 2002. doi:10.1016/s0952-1976(02)00075-1

[43] N. Xiong and L. Litz, "Reduction of fuzzy control rules by means of premise learning - method and case study," Fuzzy Sets and Systems, vol. 132, pp. 217-231, 2002. doi:http://dx.doi.org/10.1016/S0165-0114(02)00112-4

[44] S. Kamalasadan and G. Swann, "A novel power system stabilizer based on fuzzy model reference adaptive controller," in IEEE Power \& Energy Society General Meeting (PES '09'), 2009, pp. 1-8. doi:10.1109/pes.2009.5275897

[45] P. Shamsollahi and O. P. Malik, "Design of a neural adaptive power system stabilizer using dynamic back-propagation method," International Journal of Electrical Power \& Energy Systems, vol. 22, pp. 29-34, 2000 doi:10.1016/s0142-0615(99)00032-0

[46] K. R. Padiyar, Power System Dynamics Stability and Control. Hyderabad, India: BS Publications, 2008. 
[47] M. Ramirez-Gonzalez, R. Castellanos B, and O. P. Malik, "Application of simple fuzzy PSSs for power system stability enhancement of the Mexican Interconnected System," in IEEE Power and Energy Society General Meeting-2010, 2010, pp. 1-8. doi:10.1109/pes.2010.5589526
[48] F. P. Demello and C. Concordia, "Concepts of Synchronous Machine Stability as Affected by Excitation Control," IEEE Transactions on Power Apparatus and Systems, vol. PAS-88, pp. 316-329, 1969. doi:10.1109/tpas.1969.292452

\section{Biography}

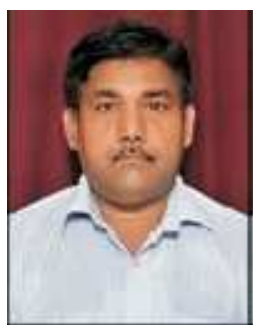

D.K. Sambariya is Associate Professor at Department of Electrical Engineering in Rajasthan Technical University, Kota (Rajasthan) India. He obtained his B.E. degree in Electrical Engineering and his M.E. degree in Power System engineering from the University of Rajasthan in 1994 and 2009, respectively.

At present, he is pursuing $\mathrm{PhD}$ from the department of Electrical Engineering, Indian Institute of Technology Roorkee, Roorkee-247667. His research interests include power system stabilizer, fuzzy logic controller, and model order reduction methods. 